\title{
A 3D Simulation approach of Pool Fire
}

\author{
Jianfeng Zhou \\ School of Electromechanical Engineering \\ Guangdong University of Technology \\ Guangzhou 510006, China. \\ Email: gzzhoujf@sina.com
}

\begin{abstract}
Pool fire is a major accident type of flammable liquid. In this paper, a particle system based 3D simulation model of pool fire flame is established. On this basis, a multipoint source model of pool fire thermal radiation is proposed. Each living particle in the particle system is considered as a radiation source. A study case of gasoline pool fire is simulated by this model, and the radiation results evaluated by this model are compared with those evaluated by the point source model and the measured data.
\end{abstract}

Keywords-Pool fire; Particle system; Multi-point source model

\section{INTRODUCTION}

Pool fire is one of the most major accidents in petrochemical industries, it accounts for a large proportion of all fire incidents. Burning pool fire flame does not have a fixed shape, but have dynamic and stochastic characteristics. This makes it difficult to use traditional three-dimensional modeling method to simulate. However, as an important virtual reality technology, particle system can easily implement fuzzy objects (fire, clouds, water, etc.) modeling. Particle system was firstly proposed in 1983 by Reeves. Its core idea is to extract rules from small physical models, and construct complex objects using simple elements [1]. After that, there have been many studies to use particle system to simulate the phenomenon of combustion [2-6].

For pool fire simulation, in addition to a realistic shape of flame, consequence simulation of the pool fire is more important, so that the impact of fire can be analyzed in the simulation environment. Thermal radiation of pool fire is the main cause of casualties and property damage. The hazard of pool fire is mainly thermal radiation damage. The establishment of thermal radiation model is very important for pool fire risk assessment. In the report [7], the main thermal radiation models of pool fire were divided into three categories: one is semi-empirical model, the other is field model, and the third is integral model. Semi-empirical models are empirical formulas based on experimental data. Field model (such as computational fluid dynamics model) solves the Navier-Stokes equations of fluid flow, needs submodels of physical and chemical processes occurring in fire to predict fire behavior. The integral model is a compromise between semi-empirical model and field model. Field model can provide more rigorous and flexible framework to solve the combustion problems than semi-empirical model. The disadvantage in using field models or integral models is that their use requires significant effort and expertise, usually the field models are time-consuming and resource consuming. The advantages of semi-empirical model are that they are relatively simple, easy to use and the predicted results are reasonable, so that semi-empirical models are more frequently used in practical risk assessments.

Three-dimensional simulation method of pool fire is studied in this paper. The 3D simulation of pool fire mainly contains the following two aspects: the first is the realistic flame; the second is the consequence of the pool fire.

\section{3D SIMULATION MODEL OF POOL FIRE}

\section{A. $3 D$ flame of pool fire}

Particle system is composed of large number of simple primitives which are called the particles. Each particle has a set of attributes such as shape, size, color, transparency, position, velocity and lifetime, etc. Particle system is not a simple static system. As time goes by, not only are the existing particles in the system kept changing, but also new particles are added to the system, some old particles will disappear. In order to simulate particle growth and death process, each particle has a certain life cycle. The pool fire flame can be simulated by particle systems.

In the simulation of flame animation sequence, each frame must perform the following steps [1]:

i. New particles are generated

ii. Each new particle is assigned its own set of attributes

iii. Any particles that have existed for a predetermined time are destroyed

iv. The remaining particles are transformed and moved according to their dynamic attributes

$\mathrm{v}$. An image of the remaining particles is rendered

For the pool fire simulation, the generation of particles, particle movement and particle extinction are introduced here.

1) Generation of pool fire particles

Assume the burning rate is equal at any point of the liquid surface. Thus, the initial positions of generated particles are uniformly distributed on the surface. Suppose the particle's position in three-dimensional space is determined by the $\mathrm{X}, \mathrm{Y}, \mathrm{Z}$ axes, and $\mathrm{Y}$-axis is the longitudinal axis which is perpendicular to the ground. For the circular liquid pool, the position of a generated particle should satisfy the following relationships: 


$$
\begin{aligned}
& x(0)=x_{0}+\operatorname{random}(-R,+R) \\
& y(0)=y_{0} \\
& z(0)=z_{0}+\operatorname{random}(-R,+R) \\
& \left(x(0)-x_{0}\right)^{2}+\left(z(0)-z_{0}\right)^{2} \leq R^{2}
\end{aligned}
$$

Where, $\mathrm{x}(0), \mathrm{y}(0), \mathrm{z}(0)$ are the initial coordinate values of a particle; $\mathrm{x} 0, \mathrm{y} 0$, and $\mathrm{z} 0$ are the coordinate values of the center point of the pool surface; $\mathrm{R}$ is the radius of the liquid pool, $\mathrm{m}$; random $(-\mathrm{R},+\mathrm{R})$ denotes a uniformly distributed random number between $-\mathrm{R}$ and $+\mathrm{R}$.

For a rectangular shape or other shapes of the liquid pool, the particle's initial position can be determined similarly.

2) Movement of pool fire particles

Pool fire flame is shown by the movement of a large number of particles. Velocity and direction of particle movement have a major impact of the pool fire shape. The velocity of a particle consists of an average and a stochastic part. The stochastic part reflects the dynamic and random character of a flame.

$$
\begin{aligned}
& u(t)=\bar{u}(t)+u^{\prime}(t) \\
& v(t)=\bar{v}(t)+v^{\prime}(t) \\
& w(t)=\bar{w}(t)+w^{\prime}(t)
\end{aligned}
$$

Where, $u(t) 、 v(t)$ 和 $w(t)$ are velocities in the X, Y and $\mathrm{Z}$ directions of a particle at the time $t$, respectively; $\bar{u}(t), \bar{v}(t), \bar{w}(t)$ are mean velocities in the $\mathrm{X}, \mathrm{Y}$ and $\mathrm{Z}$ directions of the particle at the time $t ; u^{\prime}(t), v^{\prime}(t), w^{\prime}(t)$ are stochastic velocities in the $\mathrm{X}, \mathrm{Y}$ and $\mathrm{Z}$ directions of the particle at the time $t$;

Under no wind circumstance, $\bar{u}(t)$ and $\bar{w}(t)$ are both zeros. Roaring fires will require a high particle velocity $\bar{v}(t)$, while gentle fires should comprise slow-moving particles.

It is assumed that the mean upward velocity of gases at any height $\mathrm{Y}$ is proportional to the square root of $\mathrm{Y}$. This is borne out by data from large field tests with other hydrocarbon fires where such velocity measurements have been made [8]. Thus, the mean velocity $\bar{v}(t)$ is determined as:

$$
\bar{v}(t)=\sqrt{y(t)-y(0)}
$$

Where, $y(t)$ is the $\mathrm{Y}$-axis value of the particle at time $t$.

The trajectory of each particle can be considered as a series of discrete points. By the human eye's persistence of vision, the movement of particles appears to be continuous. The discrete points of particle movement process are determined by the update intervals of the particle system. Pool fire flame is implemented by rendering all the particles at each animation frame. The time between two frames is composed of certain update intervals of the particle system. A series of animation frames can show pool fire burning flame. Particle position at time $\mathrm{t}$ can be determined as following:

$$
\begin{aligned}
& x(t)=x(t-\Delta t)+u(t) \times \Delta t \\
& y(t)=y(t-\Delta t)+v(t) \times \Delta t \\
& z(t)=z(t-\Delta t)+w(t) \times \Delta t
\end{aligned}
$$

Where, $\Delta t$ is update interval of the particle system.

3) Extinction of pool fire particles

Each particle has its lifetime. The particles die at end of their life, and they are deleted from the particle system. Particle lifetime is determined by the flame length and the velocity of the particle. As the flame mainly moves upward under no wind circumstance, so the lifetime of a particle is determined by the flame length in $\mathrm{Y}$ axis and the velocity component in $\mathrm{Y}$-axis.

One of the most well known flame length correlations is the Thomas correlation [7]:

$$
L / D=42\left[\frac{m_{b}}{\rho_{a}(g D)^{1 / 2}}\right]^{0.61}
$$

Where, $\mathrm{L}$ is the flame length, $\mathrm{m}$; $\mathrm{D}$ is the pool diameter, $\mathrm{m} ; \mathrm{m}_{\mathrm{b}}$ is the mass burning rate, $\mathrm{Kg} /\left(\mathrm{m}^{2} \mathrm{~s}\right) ; \rho_{a}$ is the ambient air density, $\mathrm{Kg} / \mathrm{m}^{3} ; \mathrm{g}$ is the acceleration due to gravity, $\mathrm{m} / \mathrm{s}^{2}$.

So, the lifetime of a particle is:

$$
\text { LifeTime }=L / \bar{v}+\tau
$$

Where, $\bar{v}$ is the mean velocity over the height 0 to $L ; \tau$ is a stochastic value.

When the flame length $L$ is determined, based on the formula (3) the mean velocity $\bar{v}$ can be determined by:

$$
\bar{v}=\frac{1}{\mathrm{~L}} \int_{y_{0}}^{L+y_{0}} \sqrt{y-y_{0}} d y=\frac{2}{3} L^{\frac{1}{2}}
$$

\section{B. Multi-point source model of thermal radiation}

In the 3D simulation, the assessment of the consequences must have rapid execution speed and use less resource due to the application needs. So, semi-empirical model is adopted under this circumstance. Most widely used semi-empirical models are the Point-source model and the Surface Emitter Model. The point source model is a correct assumption at large distances from the fire [9]. The predictions made by the point source model are on average closer to the experimental data than any other model tested in Rob Fleury's research [10]. In the particle system simulation of pool fire mentioned above, pool fire is constituted by a large number of particles. Different particles locate at different points in the flame. These particles can be directly used to represent the energy of the flame. Thus, a multi-point source model of pool fire thermal radiation can be established.

In the point-source model, the received radiation flux $\mathrm{Q}$ is given by [7]:

$$
Q=\frac{P}{4 \pi r^{2}}
$$

Where, $P$ is the emissive power of the flame, $\mathrm{kW} ; r$ is the distance from the source to the receiver, $\mathrm{m}$.

The emissive power $P$ is given by [7]: 


$$
P=m_{b} A F \Delta H_{c}
$$

Where, $m_{b}$ is the mass burning rate, $\mathrm{kg} /\left(\mathrm{m}^{2} \mathrm{~s}\right) ; A$ is the pool area, $\mathrm{m}^{2} ; F$ is the fraction of combustive energy released as radiation; $\Delta H_{c}$ is the net heat released by combustion of the fuel at its boiling point, $\mathrm{kJ} / \mathrm{kg}$.

In the particle system simulation of pool fire, the released thermal radiation of the fire can be considered as the sum of thermal radiation released by these particles. Then, each particle is looked as a radiation source. The amount of radiation received by target at time $t$ is;

$$
Q(t)=\sum_{i=1}^{K} \frac{P_{i}(t)}{4 \pi r_{i}^{2}(t)}
$$

Where, $K$ is the number of living particles in the system. Obviously, $K$ is determined by the particle system and cannot be changed by the operator freely. $P_{i}(t)$ is the emissive power of the $i$ th particle, $\mathrm{kW} ; r_{i}(t)$ the distance from the $i$ th particle to the receiver, $\mathrm{m}$. then,

Suppose the coordinates of the target is $\left(x_{t a r}, y_{t a r}, z_{t a r}\right)$,

$$
r_{i}(t)=\sqrt{\left(x_{i}(t)-x_{\text {tar }}\right)^{2}+\left(y_{i}(t)-y_{\text {tar }}\right)^{2}+\left(z_{i}(t)-z_{\text {tar }}\right)^{2}}
$$

Where, $x_{i}(t), y_{i}(t)$ and $z_{i}(t)$ are coordinates of the $i$ th particle in the $\mathrm{X}, \mathrm{Y}$ and $\mathrm{Z}$ axes at time $\mathrm{t}$;

If the released radiation of each particle is considered equal, then:

$$
P_{i}(t)=\frac{P}{K} \quad i=1,2 \ldots K
$$

\section{CASE STUDY}

Based on OpenGL, a simple 3D environment of a gasoline tank area is established, which is shown as Fig. 1. There are tanks ( $4 \mathrm{~m}$ diameter and $4 \mathrm{~m}$ height), buildings, roads and a virtual human in the scene. The main purpose of the simulation system is to analyze the response of the virtual human when an accident occurs.

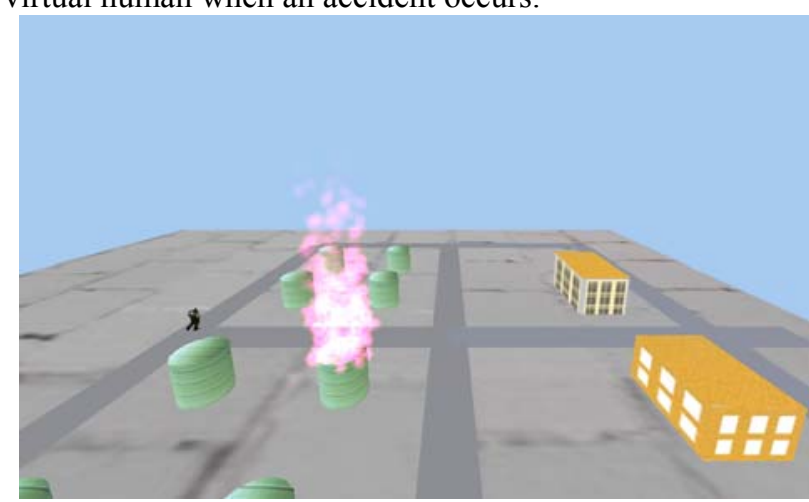

Fig.1 Particle system based gasoline pool fire simulation

When a tank fire occurs, the fire is looked as a pool fire and the possibility of explosion is not considered. The particle system based 3D simulation of the pool fire is as follows:

(1) Particle system parameters. The chances that the particles are generated on any point of the pool surface are equal. The update interval of the particle system is set to be
$3 \mathrm{~ms}$. Each update a new particle is generated. The stochastic factors describing the turbulence of a fire, such as $u^{\prime}(t), v^{\prime}(t)$, $\mathrm{w}^{\prime}(\mathrm{t})$ and $\tau$, take the normal distribution with mean 0 , and the standard deviation is $15 \%$ of the corresponding average value. They vary in a certain range only to simulate realistic fire shape, while the main structure of the flame is determined by flame length, particle average velocity, and average particle lifetime. According to the formulas (5)-(7), the flame length is $8.64 \mathrm{~m}$, the mean velocity is $2 \mathrm{~m} / \mathrm{s}$, and the mean lifetime of particles is $4.32 \mathrm{~s}$.

(2) Burning process. During the simulation, new particles emerge and old particles disappear. The number of living particles in the particle system gradually increases from zero to a steady-state, in which the number randomly varied in a limited range. In each animation frame all living particles are rendered.

(3) Thermal radiation analysis. The thermal radiation of the gasoline pool fire is analyzed by using the multi-point source model mentioned above. To compare the result with the measured radiation data, a target which is 20 meters (5 times the diameter) away from the center of the tank and in the same height with the pool surface ( $4 \mathrm{~m}$ height) is studied here. The mass burning rate is $0.055 \mathrm{Kg} /(\mathrm{m} 2 \mathrm{~s})$, and the net heat released by combustion of gasoline at its boiling point is $43700 \mathrm{KJ} / \mathrm{Kg}$ [11].

The value of $\mathrm{F}$ factor in the thermal radiation model has a great impact on determining the final amount of thermal radiation. Generally, a larger $\mathrm{F}$ value corresponds to a smaller diameter of the liquid pool. Table 1 shows the Ffactor values for gasoline point-source model, derived from external heat flux measurement [7].

Table $1 \mathrm{~F}$ factors derived from radiation measurement from gasoline pool fires

\begin{tabular}{|c|c|c|}
\hline Fuel & Pool diameter $(\mathrm{m})$ & F factor $(\%)$ \\
\hline gasoline & 1 & 40 \\
\hline gasoline & 6 & 15 \\
\hline gasoline & 10 & 8 \\
\hline gasoline & 20 & 7 \\
\hline
\end{tabular}

In this case studying, the $\mathrm{F}$ factor value is taken as $25 \%$. After the simulation goes into the steady state, the thermal radiation flux at the target is sampled. Ten samples and corresponding living particles are shown in Table 2.

The average of the above 10 radiation flux samples is $1.44 \mathrm{~kW} / \mathrm{m}^{2}$. The radiation flux calculated by point-source model at the target is $1.577 \mathrm{~kW} / \mathrm{m}^{2}$. Under the same circumstance, the observed mean radiation value is approximately $1.4 \mathrm{~kW} / \mathrm{m}^{2}[12]$

To validate the results of multi-point source model with other published data [12], the radiation evaluated at the dimensionless distance of five diameters is also presented in this study. The comparisons of gasoline pool fires are shown as Fig.2. Three diameters which are $1.5 \mathrm{~m}, 3 \mathrm{~m}$ and $4 \mathrm{~m}$ are studied.

Table 2 Radiation flux samples at the target

\begin{tabular}{|c|c|c|}
\hline No. & Living particles & Radiation flux $\left(\mathrm{kW} / \mathrm{m}^{2}\right)$ \\
\hline 1 & 493 & 1.4461 \\
\hline 2 & 509 & 1.4426 \\
\hline
\end{tabular}




\begin{tabular}{|c|c|c|}
\hline 3 & 515 & 1.4388 \\
\hline 4 & 515 & 1.4371 \\
\hline 5 & 517 & 1.4325 \\
\hline 6 & 507 & 1.4381 \\
\hline 7 & 510 & 1.4406 \\
\hline 8 & 509 & 1.4390 \\
\hline 9 & 515 & 1.4378 \\
\hline 10 & 515 & 1.4416 \\
\hline
\end{tabular}

The results show that based on particle system 3D simulation of pool fire, the multi-point source model established by directly using the particles can reflect the thermal radiation well. The radiation evaluated by the multipoint source model is better than that evaluated by the point source model.

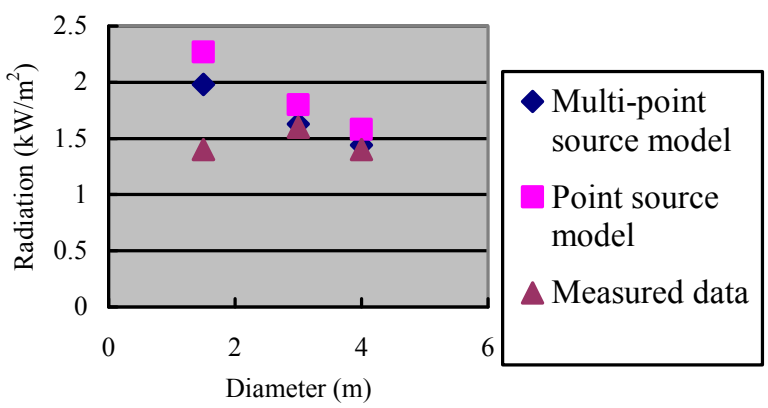

Fig. 2 Comparison of gasoline pool fire radiation

The comparisons of diesel pool fire are shown as Fig. 3 . Four diameters which are $1.5 \mathrm{~m}, 3 \mathrm{~m}, 4 \mathrm{~m}$ and $6 \mathrm{~m}$ are studied.

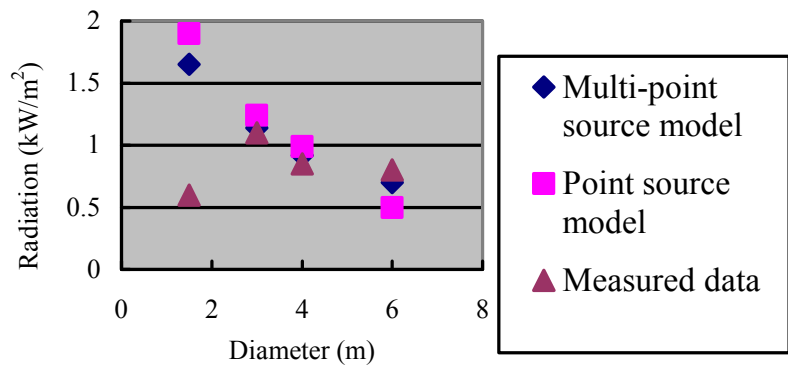

Fig.3 Comparison of diesel pool fire radiation

\section{CONCLUSIONS}

For flammable liquid pool fire, a three-dimensional simulation model is established. The model includes two aspects: one is the flame simulation of pool fire; the other is the thermal radiation model.

The flame simulation model is established according to particle systems. The flame is considered as the movement of a large number of particles. The turbulence of the flame is simulated by Monte Carlo method. The attributes of the particles such as position, velocity and lifetime are kept changing during the movement.

Based on the particle system simulation of pool fire, the living particles are considered as radiation sources, so that a multi-point source model of pool fire thermal radiation is established. Real burning flame will emit thermal power from all parts of the flame.

\section{ACKNOWLEDGMENT}

This work is supported by Natural Science Foundation of Guangdong Province in China (No. S2011040003990).

\section{REFERENCES}

[1] W. T. Reeves, "Particle Systems - A Technique for Modeling a Class of Fuzzy Objects," ACM Transactions on Graphics, Vol. 2, pp.91-108, 1983.

[2] S. Zhou, Y. Sun, L. Lu and Z. Chen, "Fire Simulation Model Based on Particle System and Its Application in Virtual Reality," Proceedings of the 16th International Conference on Artificial Reality and Telexistence--Workshops (ICAT'06), Hangzhou, China, 2006.

[3] B. Philippe, P. Sébastien and P. Pierre, "Realistic and controllable fire simulation," Proceedings of Graphics interface, Ottawa, Ontario, Canada, 2001.

[4] X. Wei, W. Li and K. Mueller, et al., "Simulating Fire with Texture Splats," Proceedings of the conference on Visualization '02, Boston, Massachusetts, USA, 2002.

[5] A. Lamorlette and N. Foster, "Structural Modeling of Flames for a Production Environment," Proceedings of the 29th annual conference on Computer Graphics and Interactive Techniques, San Antonio, Texas, USA, 2002.

[6] H. Lee, L. Kim and M. Meyer, et al., "Meshes on Fire, " EG Workshop on Computer Animation and Simulation, Manchester, U.K., 2001.

[7] L.T. Cowley and A.D. Johnson, "Oil and gas fires: characteristics and impact," http://www.hse.gov.uk/research/otipdf/oti92596.pdf, 1992.

[8] P. K. Raj, "Large hydrocarbon fuel pool fires: Physical characteristics and thermal emission variations with height," Journal of Hazardous Materials, Vol.140, pp. 280-292, 2007

[9] Society of Fire Protection Engineers, Engineering Guide: Assessing Flame Radiation to External Targets from Pool Fires, Society of Fire Protection Engineers, Bethesda, Maryland, 1999.

[10] R. Fleury, "Evaluation of Thermal Radiation Models for Fire Spread Between Objects," http://ir.canterbury.ac.nz/bitstream/10092/4959/1/ thesis_fulltext.pdf, 2010.

[11] U.S. Nuclear Regulatory Commission, "Fire Dynamics Tools (FDTs) Quantitative Fire Hazard Analysis Methods for the U.S. Nuclear Regulatory Commission Fire Protection Inspection Program (NUREG-1805, Final Report)," http://www.nrc.gov/reading$\mathrm{rm} /$ doccollections/nuregs/ staff/sr1805/final-report/, 2004.

[12] M. Munoz, J. Arnaldos and J. Casal, et al., "Analysis of the geometric and radiative characteristics of hydrocarbon pool fires," Combustion and Flame, Vol. 139, pp. 263-277, 2004. 